\title{
The Association of Perceived Neighborhood Walkability and Environmental Pollution With Frailty Among Community-dwelling Older Adults in Korean Rural Areas: A Cross-sectional Study
}

\author{
Mi-Ji Kim', Sung-Hyo Seo', Ae-Rim Seo', Bo-Kyoung Kim', Gyeong-Ye Lee², Yeun-Soon Choi', Jin-Hwan Kim³, \\ Jang-Rak Kim', Yune-Sik Kang ', Baek-Geun Jeong', Ki-Soo Park ${ }^{1,2}$ \\ ${ }^{1}$ Department of Preventive Medicine and Institute of Health Science, Gyeongsang National University College of Medicine, Jinju, Korea; ${ }^{2}$ Center for \\ Farmer's Safety and Health, Gyeongsang National University Hospital, Jinju, Korea; ${ }^{3}$ Department of Health Policy and Management, Seoul National \\ University College of Medicine, Seoul, Korea
}

Objectives: The aim of this study was to evaluate the associations of frailty with perceived neighborhood walkability and environmental pollution among community-dwelling older adults in rural areas.

Methods: The participants were 808 community-dwelling men and women aged 65 years and older in 2 rural towns. Comprehensive information, including demographics, socioeconomic status, grip strength, polypharmacy, perceived neighborhood environment (specifically, walkability and environmental pollution), and frailty, was collected from participants using face-to-face interviews conducted between June and August 2018. Perceived neighborhood walkability was measured using 20 items that were selected and revised from the Neighborhood Environment Walkability Scale, the Neighborhood Walkability Checklist from the National Heart Foundation of Australia, and the Physical Activity Neighborhood Environment Survey. The Kaigo-Yobo Checklist was used to assess participants' frailty.

Results: The overall prevalence of frailty in this community-dwelling population was $35.5 \%$. Sex, age, cohabitation status, educational attainment, employment status, grip strength, and polypharmacy were significantly associated with frailty. In the logistic regression analysis, frailty was associated with low perceived neighborhood walkability (adjusted odds ratio [aOR], 0.881; 95\% confidence interval $[\mathrm{Cl}], 0.833$ to $0.932 ; p<0.001$ ) and severe perceived neighborhood environmental pollution ( $\mathrm{aOR}, 1.052 ; 95 \% \mathrm{Cl}, 1.017$ to 1.087 ; $p=0.003$ ) after adjusting for sex, age, cohabitation status, educational attainment, employment status, monthly income, grip strength, and polypharmacy.

Conclusions: More studies are warranted to establish causal relationships between walkability and environmental pollution and frailty.

Key words: Frailty, Built environment, Environmental pollution, Rural population, Frail elderly, Korea

Received: June 26, 2019 Accepted: October 24, 2019

Corresponding author: Ki-Soo Park, MD, PhD

Department of Preventive Medicine and Institute of Health Science, Gyeongsang National University College of Medicine, 15 Jinju-daero 816beon-gil, Jinju 52727, Korea

E-mail: parkks@gnu.ac.kr

This is an Open Access article distributed under the terms of the Creative Commons Attribution Non-Commercial License (http://creativecommons.org/licenses/bync/4.0// which permits unrestricted non-commercial use, distribution, and reproduction in any medium, provided the original work is properly cited.

\section{INTRODUCTION}

According to 2017 data from Statistics Korea, Korea's elderly population, 65 and older, accounted for $13.9 \%$ of the entire population, making the country an aging society. Korea is expected to become a super-aged society and the proportion of the elderly population will grow to $24.5 \%$ of the entire population by 2030 [1]. 
The growth of the elderly population in Korea sheds light on the concept of frailty. Frailty is a dynamic state that involves losses in one or more domains of human functioning (physical, psychological, or social). Frailty is caused by a wide range of factors and increases the risk of adverse outcomes, such as hospitalization, falling, and death [2]. Research on frailty is crucial because frailty is reversible with appropriate interventions to mitigate risk factors before it reaches an irreversible phase of disability or death. Such interventions can therefore prevent the disability or death of elderly individuals and help them maintain their physical, psychological, and social functioning [3].

The determinants of frailty include daily habits, such as diet, smoking, and drinking; genetic factors; socioeconomic status, such as educational attainment and income; and demographics, such as age, sex, marital status, and ethnicity. Characteristics of the individual's residential environment like neighborhood safety and life events like the loss of one's spouse may also impact frailty. Morbidity also plays a role in earlier frailty [4]. Nutrition, mobility, physical activity (PA), muscle strength, endurance, sense of balance, and sensory functioning are determinants of physical frailty, while psychological frailty is associated with factors such as cognitive function, depression, and coping ability, and social frailty with social relationships and support [2].

Interventions to increase PA and socialization are important to reduce frailty among the elderly [5]. However, few studies have examined the effects of an individual's local environment on their level of PA, social involvement, and accessibility. A study by Xue et al. [6] found that environmental factors such as one's built environment can be determinants of frailty, but few follow-up research has been conducted to support this idea.

A recent systematic review of the literature suggests that the level of PA is associated with the local environment. The built environment includes access to destinations and services, safety of individuals against crime, access to stores, a green and aesthetically pleasing view, a walkable social infrastructure (as measured by Neighborhood Environment Walkability Scale; NEWS), and access to public transportation. The built environment was strongly associated with PAs including walking of the elderly aged 65 and older and the trends of association were similar when measured by both objective and subjective standards [7]. Parts of the built environment, such as parks and community gardens, were found to lead to increased PA and social connectedness among the elderly [8]. A good built envi- ronment in the community promotes social activity among senior citizens, ultimately affecting their health, as retired senior citizens spend much of the day within their communities [9]. Natural environments, such as air quality and noise pollution, can also impact the health of senior citizens by causing cardiovascular and respiratory diseases [10,11].

Previous studies have proved how the local environment can affect various aspects of the health status of the elderly, including PAs and social relationships. However, no research has been conducted on how the environment may lead to frailty internationally, particularly in the context of rural areas. Barnett et al. [7] reported in their systematic review that only 3 out of 100 studies focusing on the relationship between the local environment and PA among the elderly were conducted in a rural environment. As rural areas tend to have a higher proportion of aged individuals than cities and also have an inferior built environment; it makes sense to carry out additional research on rural areas.

This study was conducted to investigate correlations between perceived neighborhood walkability, environmental pollution, and frailty, thereby generating data on how to prevent negative health results caused by frailty in rural areas and contributing to the promotion of elderly health.

\section{METHODS}

\section{Study Design and Setting}

This cross-sectional study is a part of the broader research agenda aiming to identify determinants of walking exercise among residents of rural towns. This study was conducted among adults aged 65 and older who were participants of the parent study. The study took place in 2 myeons (a myeon is an administrative unit in Korea similar to a township) of Gyeongnam Province. The towns in this study are rural, mainly producing rice and watermelons, and have been designated as targets for the Resident-engaged Health Promotion Program of Gyeongnam Province. Six interviewers received 2 training sessions regarding content and data collection by the research team, and these interviewers carried out the survey for 2 months starting in June 2018. Data collection was performed via 1-on1 interviews, during which the interviewer read out structured survey questions and the subject responded. The interviewer also took physical measurements. Personal data were protected by coding and anonymizing identifiable information, including names, and study materials will be kept in locked stor- 
age after completion of the study and will be disposed of after 3 years. Written consent for voluntary participation was obtained after the researchers provided the participants with adequate information on the purpose and methods, potential inconveniences and benefits, protection and management of personal data, the right to refuse participation without penalty, and the right to withdraw consents to participate.

\section{Participants}

Participants were adults aged 19 and older residing in the 2 towns of Gyeongnam Province. Interviewers visited households with even-numbered road name addresses and interviewed all members of those households aged 19 and older. Adults aged 65 and older were additionally assessed for their frailty and grip strength. In cases where an address was unoccupied (empty homes, greenhouses, or etc.), a special facility (orphanages, elderly homes, dormitories, temples, convents, inns, hotels, or etc.), or occupied by foreign nationals, or where no occupants during 3 visits, it was replaced by the next oddnumbered address. The number of optimal participants was determined to be 1450 , which was calculated at a $95 \%$ confidence level using a $\pm 2.1 \%$ p sampling error of the population size of 5700, and the actual number of participants for the survey was set at 1500 to prepare for the potential missing values in the survey data. All individuals who could understand verbalized questions and deliberately respond by speaking, and who signed an agreement to participate were selected as subjects. Among the 1500 final participants, 817 were aged 65 and older. Of these, 808 (331 males and 477 females) were included in the final analysis in this study; the remaining 9 participants were excluded due to missing values in their responses.

\section{Variables and Measurement}

The exposure variables of this study were perceived neighborhood walkability and environmental pollution. The outcome variable was frailty. The covariates included demographics (sex, age, and cohabitation status), socioeconomic status (educational attainment, occupation, and average monthly income), grip strength, and polypharmacy.

Recruiting voluntary participants through the local community health center or town office is likely to disproportionately attract people who are relatively healthy, socially active, and highly educated. To avoid such selection bias, this survey was conducted among all adults who were accessible when interviewers visited buildings with even-numbered road name ad- dresses. The research team trained the interviewers to perform interviews according to the pre-set guideline, and we also monitored the interviews occasionally. If an interview could not be performed at a certain address, interviewers were allowed to move on to the next address after discussing the situation with the research team. Participation was encouraged by notifying the residents of the visit via local community health centers, such that the final participants represented a high percentage of the entire pool of potential study subjects. The 6 interviewers had 2 education and training sessions with structured survey questions and detailed guidelines to prevent biases such as interviewer bias and measurement bias. The potential for confirmation bias was controlled by hiring interviewers who were not aware of the hypothesis of the study to carry out data collection on behalf of the research team. The impact of confounding bias was minimized by collecting data regarding potential confounding variables, such as demographic and socioeconomic characteristics and physical health status. The impact of severe cognitive disabilities that may influence the frailty was controlled by limiting participants to individuals who could understand verbalized questions and deliberately respond by speaking.

\section{Outcome variables}

\section{Frailty (the Kaigo-Yobo Checklist)}

Frailty was measured using the Korean version of the KaigoYobo Checklist. This tool was developed by the Tokyo Metropolitan Institute of Gerontology and consists of 15 self-report questions, including 4 on nutritional state, 2 on falling accidents, 2 on getting out of the home, 2 on social relationships and support, and 1 each on hobbies, mobility, vision (as a communication ability), and general health status (Supplemental Material 1). The validity and credibility of this survey have been confirmed for Korean senior citizens [12]. The aggregate score ranged from 0 to 15 . The higher the total score, the frailer the respondent. Frailty was determined in accordance with the standard set by Murayama et al. [13]: scores under 4 were defined as not frail, while scores of 4 and higher were defined as frail.

\section{Exposure variables}

Perceived neighborhood walkability

The research team reorganized existing survey questions used to measure perceived neighborhood walkability to better represent domestic rural environments. Questions were 
designed with reference to the NEWS (self-report format, 98 questions), which is the most widely used tool in the world [14], as well as the Neighborhood Walkability Checklist (self-report format, 30 questions) [15] provided by the National Heart Foundation of Australia and the Physical Activity Neighborhood Environment Survey (International Physical Activity Prevalence Study/International Physical Activity Questionnaire Environmental Survey Module; self-report format, 17 questions) [16] developed by the International Physical Activity Prevalence Study. The final survey tool used in this study consisted of a total of 20 self-report questions (Supplementl Material 2). The survey measured walkability across 4 domains, including pedestrian friendliness (e.g., impediments to pedestrians and street aesthetics), comfort (e.g., the presence of flat and wide streets, rest areas, and restrooms), safety (e.g., nighttime lighting and separation of sidewalks from roads), and convenience (e.g., the presence of signs and accessibility to stores and parks). Each domain had 5 yes-or-no questions; participants were given 1 point for "yes" and 0 points for "no," except for the first question in the pedestrian friendliness domain, which was allocated 0 points for "yes" and 1 point for "no."The overall evaluation was performed using aggregate scores, which ranged from 0 points to 5 points per domain and from 0 to 20 for all items. The higher the total score, the better the perceived neighborhood walkability in the area. The internal consistency reliability of the tool as confirmed by Cronbach's $\alpha$ coefficient was 0.675 , implying acceptable internal consistency.

\section{Perceived neighborhood environmental pollution}

A self-report survey consisting of 7 questions was developed by the research team to evaluate perceived neighborhood environmental pollution in Korean rural areas (Supplemental Material 3). The survey items were based on the 2015 study by Hanibuchi et al. [17], which focused on natural environmental factors that may impact PA, particularly walking. The survey used in the study included 3 questions on air pollution, 1 on water pollution, and 1 on noise pollution, as well as 2 questions on temperature and excessive sunlight. In our study, the tool asked the respondents to evaluate the degree of environmental pollution in the main areas where they walk, with options of "not severe (0 points)", "less severe (1 point)", "moderate (2 points)", "severe (3 points)", or "very severe (4 points)" for each question. The total score was between 0 and 28 , with higher scores indicating more severe the perceived neighborhood environmental pollution.

\section{Covariates}

Demographic and socioeconomic characteristics

Demographics included sex (male or female), age, and cohabitation status (living alone, with a spouse, with a spouse and other family members, or with non-family cohabitants). Socioeconomic status included educational attainment (none, elementary school, middle school, high school, or college and up [including community college]), occupation (manager, professional and related jobs, office worker, service worker, salesperson, agriculture/forestry/fishery worker, technician and related jobs, mechanic, machine operation and assembly worker, labor worker, career military officer, student, housekeeper, or unemployed), and income, measured as average monthly income over the past year including pension, benefits, and financial support from family (less than 1 million Korean won [KRW], 1-2 million KRW, 2-3 million KRW, 3-4 million KRW, 4-5 million KRW, or more than 5 million KRW). Sex was analyzed as a binary variable (male or female), age as a continuous variable, cohabitation status as a binary variable (yes or no), educational attainment as an ordinal variable (none [0 years], elementary [ $\leq 6$ years], or middle school and up [ $>6$ years]), occupation as a binary variable (yes or no; housekeepers were considered to have no occupation), and monthly income as a binary variable (less than 2 million KRW or 2 million KRW or more), where the threshold for monthly income was based on the minimum living expenses for two-person households in 2018 [18].

\section{Grip strength and polypharmacy}

Grip strength and polypharmacy were included in the survey to identify participants' multimorbidity and sarcopenia, which may relate to frailty. Grip strength was measured as a surrogate index of sarcopenia in this study. Measuring grip strength is one of the simplest and least invasive methods available to estimate the skeletal muscle mass of the entire body [19]. Grip strength was measured with a digital grip force meter (TKK 5401; Takei Scientific Instruments Co. Ltd., Tokyo, Japan). Participants were asked to grip the meter as hard as possible starting with their dominant hand in a straight standing position with their arms at their sides. The interviewers asked the participants to maintain this position as the measurement was taken. The participants gripped the meter for a minimum of 60 seconds before switching hands to allow for recovery of muscle strength. Measurements were taken twice for each hand, and the best result obtained with the dominant hand 
was recorded as the final value. Participants with conditions that make grip strength measurement difficult, including amputation of the arm, hand, or thumb; a cast on the hand or wrist; surgery on the hand and wrist area within the past 3 months; or pain, ache, or stiffness in the hand or wrist area within the past week, were excluded from the measurements. Poor grip strength was defined according to the Asian Working Group for Sacrgopenia: under $26 \mathrm{~kg}$ for males and under $18 \mathrm{~kg}$ for females [20]. Polypharmacy was investigated to reflect the multimorbidity of chronic diseases. Participants were asked to answer "yes" or "no" to the question "Do you take 3 or more medications daily?" Respondents who replied "yes" were categorized in the "polypharmacy" group. Temporary medications for colds or bruising and nutritional supplements were not counted, nor were medications that were taken irregularly.

\section{Statistical Analysis}

The chi-squared test was used to analyze the categorical variables. The continuous variables were analyzed with the independent $t$-test and the Wilcoxon rank-sum test to compare frail and non-frail individuals with regard to demographics, socioeconomic status, grip strength, polypharmacy, and the perceived neighborhood walkability and environmental pollution. Logistic regression analysis was performed to determine the correlations between frailty, walkability, and environmental pollution after controlling for the adjusted variables (sex, age, cohabitation status, educational attainment, occupation, monthly income, grip strength, and polypharmacy). Logistic regression analysis was performed in 2 models. The unadjusted model utilized multiple logistic regression with only 2 exposure variables (perceived neighborhood walkability and environmental pollution), while the final analysis of the adjusted model was performed using multiple logistic regression, accounting for sex, age, cohabitation status, educational attainment, occupation, monthly income, grip strength, and polypharmacy. In the multiple logistic regressions, variables were selected using the input method, the fitness of the model was confirmed with the Pearson goodness-of-fit test, and the explainability of the model was checked with Cox and Snell's coefficient of determination. The multicollinearity among the variables in the model was tested using the variance inflation factor. For the two-tailed test, $p$-values under 0.05 were considered to indicate statistical significance, and all statistical analyses were performed with SAS version 9.4 (SAS Institute Inc., Cary, NC, USA).

\section{Ethics Statement}

The study was conducted with the approval of the Institutional Review Board of Gyeongsang National University (GIRBA18-Y-0027).

\section{RESULTS}

\section{Demographic and Socioeconomic Characteristics,} Grip Strength, and Polypharmacy of Subjects

The average age of the 808 participants was $74.58 \pm 6.26$ years old, and the group consisted of 477 females (59.0\%) and 271 males (33.5\%). With regard to educational attainment, 259 (32.1\%) of the participants had received no formal education, $337(41.8 \%)$ had received 6 years or fewer of education, and $212(26.2 \%)$ had received more than 6 years of education. Regarding occupation, 401 (49.6\%) had no occupation or were housewives, and 717 (88.7\%) earned a monthly income of less than 2 million KRW. Additionally, 323 (40.0\%) had low grip strength, and 276 (34.2\%) were taking multiple medications.

In total, $35.5 \%(n=287)$ of the 808 participants were frail. The average ages of the frail and non-frail participants were $77.33 \pm 6.26$ and $73.06 \pm 5.72$, respectively, and the higher age of the frail participants was statistically significant $(p<0.001)$. Females had a higher proportion of frailty at $39.2 \%$ $(n=187)$ than males at $30.2 \%(n=100 ; p=0.009)$. Those who lived alone had a higher frailty rate $(42.1 \%, \mathrm{n}=114)$ than those who lived with someone else $(32.2 \%, \mathrm{n}=173 ; p=0.006)$. Frailty was more common among those with no formal education $(52.5 \%, \mathrm{n}=136)$ than among those with 6 years or fewer of education $(30.6 \%, n=103)$ or more than 6 years of education $(22.6 \%, \mathrm{n}=48 ; p<0.001)$. Frailty was more common among those without an occupation $(48.1 \%, \mathrm{n}=193)$ than among those with an occupation $(23.1 \%, \mathrm{n}=94 ; p<0.001)$. No significant difference in the prevalence of frailty by monthly income level was detected $(p=0.440)$. Frailty was more common among participants with low grip strength $(52.3 \%, \mathrm{n}=169)$ than among those with robust grip strength $(24.3 \%, \mathrm{n}=118 ; p<0.001)$, and participants with polypharmacy were more likely to be frail $(50.7 \%, n=140)$ than those without polypharmacy $(27.6 \%, n=$ 147; $p<0.001$ ) (Table 1).

\section{Perceived Neighborhood Walkability and Environmental Pollution}

The average score for perceived neighborhood walkability was $11.81 \pm 3.16$ points, pedestrian friendliness $3.45 \pm 1.04$ 
Table 1. Demographic and socioeconomic characteristics, grip strength, and polypharmacy of subjects

\begin{tabular}{|c|c|c|c|c|}
\hline Characteristics & $\begin{array}{c}\text { Total } \\
(n=808)\end{array}$ & $\begin{array}{c}\text { Frail } \\
(n=287)\end{array}$ & $\begin{array}{c}\text { Non-frail } \\
(n=521)\end{array}$ & $p$-value ${ }^{1}$ \\
\hline \multicolumn{5}{|l|}{ Sex } \\
\hline Male & $331(41.0)$ & $100(30.2)$ & $231(69.8)$ & 0.009 \\
\hline Female & $477(59.0)$ & $187(39.2)$ & $290(60.8)$ & \\
\hline Age, mean $\pm S D(y)$ & $74.58 \pm 6.26$ & $77.33 \pm 6.26$ & $73.06 \pm 5.72$ & $<0.001^{2}$ \\
\hline \multicolumn{5}{|l|}{ Cohabitation status } \\
\hline Living alone & $271(33.5)$ & $114(42.1)$ & $157(57.9)$ & 0.006 \\
\hline Living together & $537(66.5)$ & $173(32.2)$ & 364 (67.8) & \\
\hline \multicolumn{5}{|l|}{ Education level (y) } \\
\hline None & $259(32.1)$ & $136(52.5)$ & $123(47.5)$ & $<0.001$ \\
\hline$\leq 6$ & $337(41.8)$ & $103(30.6)$ & $234(69.4)$ & \\
\hline$>6$ & $212(26.2)$ & $48(22.6)$ & $164(77.4)$ & \\
\hline \multicolumn{5}{|l|}{ Job } \\
\hline Unemployed & $401(49.6)$ & $193(48.1)$ & $208(51.9)$ & $<0.001$ \\
\hline Employed & $407(50.4)$ & $94(23.1)$ & $313(76.9)$ & \\
\hline \multicolumn{5}{|c|}{ Monthly income (million KRW) } \\
\hline$<2$ & 717 (88.7) & $258(36.0)$ & $459(64.0)$ & 0.440 \\
\hline$\geq 2$ & $91(11.3)$ & $29(31.9)$ & $62(68.1)$ & \\
\hline \multicolumn{5}{|l|}{ Grip strength } \\
\hline Poor & $323(40.0)$ & $169(52.3)$ & $154(47.7)$ & $<0.001$ \\
\hline Robust & $485(60.0)$ & $118(24.3)$ & $367(75.7)$ & \\
\hline \multicolumn{5}{|l|}{ Polypharmacy } \\
\hline Yes & $276(34.2)$ & $140(50.7)$ & $136(49.3)$ & $<0.001$ \\
\hline No & 532 (65.8) & $147(27.6)$ & $385(72.4)$ & \\
\hline
\end{tabular}

Values are presented as number (\%).

SD, standard deviation; KRW, Korean won.

'Student's t-test.

${ }^{2}$ Chi-squared test.

points, comfort $2.40 \pm 1.05$ points, safety $3.16 \pm 1.29$ points, and convenience $2.80 \pm 1.23$ points. The total scores for perceived neighborhood walkability were lower among frail participants, with an average of $11.06 \pm 3.08$, than among nonfrail participants, who had an average score of $12.22 \pm 3.13$ $(p<0.001)$. The frail participants had a lower average score for safety ( $2.91 \pm 1.23$ points) than the non-frail participants (3.31 \pm 1.30 points; $p<0.001)$; additionally, the frail participants gave lower convenience score ( $2.38 \pm 1.28$ points) than the non-frail participants, who gave average scores of $3.03 \pm$ 1.15 points $(p<0.001)$.

The median (interquartile range; IQR) total score for perceived neighborhood environmental pollution among subjects was 10 (IQR, 7 to 14) points, with each domain scoring 1 (IQR, 0 to 2) point for air pollution category 1 (vehicle exhaust fumes), 1 (IQR, 0 to 2 ) point for air pollution category 2 (partic-
Table 2. Perceived neighborhood walkability and environmental pollution

\begin{tabular}{|c|c|c|c|c|}
\hline Variables & $\begin{array}{c}\text { Total } \\
(\mathrm{n}=\mathbf{8 0 8})\end{array}$ & $\begin{array}{c}\text { Frail } \\
(n=287)\end{array}$ & $\begin{array}{c}\text { Non-frail } \\
(n=521)\end{array}$ & $p$-value ${ }^{1}$ \\
\hline \multicolumn{5}{|c|}{ Perceived neighborhood walkability, mean \pm SD } \\
\hline Pedestrian friendliness & $3.45 \pm 1.04$ & $3.42 \pm 1.04$ & $3.46 \pm 1.03$ & 0.626 \\
\hline Comfort & $2.40 \pm 1.05$ & $2.36 \pm 1.03$ & $2.42 \pm 1.06$ & 0.414 \\
\hline Safety & $3.16 \pm 1.29$ & $2.91 \pm 1.23$ & $3.31 \pm 1.30$ & $<0.001$ \\
\hline Convenience & $2.80 \pm 1.23$ & $2.38 \pm 1.28$ & $3.03 \pm 1.15$ & $<0.001$ \\
\hline Total & $11.81 \pm 3.16$ & $11.06 \pm 3.08$ & $12.22 \pm 3.13$ & $<0.001$ \\
\hline \multicolumn{5}{|c|}{ Perceived neighborhood environmental pollution, median (IQR) } \\
\hline Air pollution $1^{2}$ & $1(0,2)$ & $1(0,2)$ & $1(0,2)$ & 0.070 \\
\hline Air pollution $2^{3}$ & $1(0,2)$ & $2(0,3)$ & $1(0,2)$ & 0.035 \\
\hline Air pollution $3^{4}$ & $2(0,3)$ & $2(0,3)$ & $2(0,3)$ & 0.145 \\
\hline Water pollution & $0(0,2)$ & $0(0,2)$ & $0(0,2)$ & 0.148 \\
\hline Noise pollution & $0(0,1)$ & $0(0,2)$ & $0(0,1)$ & 0.072 \\
\hline Excessive sunlight & $3(1,4)$ & $3(2,4)$ & $3(0,4)$ & 0.002 \\
\hline Heat or cold wave & $3(1,3)$ & $3(2,4)$ & $2(0,3)$ & 0.001 \\
\hline Total & $10(7,14)$ & $11(8,15)$ & $10(6,14)$ & 0.001 \\
\hline
\end{tabular}

SD, standard deviation; IQR, interquartile range.

${ }^{1} S$ tudent's $t$-test for perceived neighborhood walkability; Wilcoxon rank-sum test for perceived neighborhood environmental pollution.

${ }^{2}$ Air pollution 1 included vehicle exhaust fumes.

${ }^{3}$ Air pollution 2 included particulate matter.

${ }^{4}$ Air pollution 3 included livestock waste, garbage, pesticide, fertilizer, and industrial emissions.

ulate matter), 2 (IQR, 0 to 3 ) points for air pollution category 3 (livestock waste, garbage, pesticide, fertilizer, and industrial emissions), 0 (IQR, 0 to 2) points for water pollution, 0 (IQR, 0 to 1) points for noise pollution, 3 (IQR, 1 to 4 ) points for excessive sunlight, and 3 (IQR, 1 to 3 ) points for temperature. The total score for perceived neighborhood environmental pollution was higher among the frail participants, at 11 (IQR, 8 to 15) points, compared to 10 (IQR, 6 to 14) points among the non-frail participants $(p=0.001)$. Air pollution due to particulate matter scored 2 (IQR, 0 to 3 ) points in the frail participants compared to 1 (IQR, 0 to 2 ) point in the non-frail participants ( $p=0.035)$, Excessive sunlight scored 3 (IQR, 2 to 4 ) points in the frail participants and 3 (IQR, 0 to 4$)$ points in the non-frail participants ( $p=0.002)$, and temperature scored 3 (IQR, 2 to 4$)$ points in the frail participants and 2 (IQR, 0 to 3 ) points in the non-frail participants $(p=0.001)$, demonstrating statistical significance (Table 2).

\section{Perceived Neighborhood Walkability and Environmental Pollution and Frailty}

Multiple logistic regression analysis was performed to identify correlations between frailty and perceived neighborhood 
Table 3. Logistic regression results of frailty according to perceived neighborhood walkability and environmental pollution

\begin{tabular}{llccc}
\hline Variables & \multicolumn{1}{c}{ Unadjusted } & p-value & Adjusted $^{1}$ & $\boldsymbol{p}_{\text {-value }}$ \\
\hline Walkability $^{2}$ & $0.898(0.856,0.942)$ & $<0.001$ & $0.881(0.833,0.932)$ & $<0.001$ \\
Environmental pollution $^{3}$ & $1.034(1.005,1.063)$ & 0.021 & $1.052(1.017,1.087)$ \\
Cox and Snell R $^{2}$ & $0.051(<0.001)$ & & $0.306(<0.001)$
\end{tabular}

Values are presented as odds ratio (95\% confidence interval).

'The adjusted model controls for sex (male $=0$, female $=1$ ), age (continuous), cohabitation status (living alone $=1$, living together $=0$ ), education level (none, $\leq 6 \mathrm{y}$, $>6 \mathrm{y}=$ reference), employment status (unemployed=1, employed=0), monthly income $(<2$ million Korean won $[\mathrm{KRW}]=1, \geq 2$ million $\mathrm{KRW}=0)$, grip strength $($ poor $=1$, robust $=0)$, and polypharmacy (yes $=1$, no $=0$ ).

${ }^{2}$ Perceived neighborhood walkability.

${ }^{3}$ Perceived neighborhood environmental pollution.

walkability and environmental pollution. In the unadjusted model, perceived neighborhood walkability (odds ratio [OR], $0.898 ; 95 \%$ confidence interval $[\mathrm{Cl}], 0.856$ to $0.942 ; p<0.001$ ) and perceived neighborhood environmental pollution (OR, $1.034 ; 95 \% \mathrm{Cl}, 1.005$ to $1.063 ; p=0.021)$ were associated with frailty. Perceived neighborhood walkability (OR, $0.881 ; 95 \% \mathrm{Cl}$, 0.833 to $0.932 ; p<0.001$ ) and perceived neighborhood environmental pollution (OR, $1.052 ; 95 \% \mathrm{Cl}, 1.017$ to 1.087 ; $p=0.003$ ) were associated with frailty in the adjusted model, which adjusted for sex, age, cohabitation status, educational attainment, occupation, income, grip strength, and polypharmacy. In other words, frailty was more common in environments with lower perceived neighborhood walkability and higher perceived neighborhood environmental pollution. The Cox and Snell $R^{2}$ value of the unadjusted model was 0.051 $(p<0.001)$, and the value was higher in the adjusted model $(0.306 ; p<0.001)$ in the adjusted model (Table 3$)$.

\section{DISCUSSION}

This study used a self-report survey tool to measure perceived neighborhood walkability and environmental pollution to identify correlations between the perceived local environment and frailty in Korean rural residents. Frailty was evaluated using the Kaigo-Yobo Checklist. The results showed that lower perceived neighborhood walkability and a higher level of perceived neighborhood environmental pollution were associated with a higher level of frailty after controlling for potential confounders.

This study found a frailty prevalence of $35.5 \%$ among rural residents aged 65 and older. Previous studies have suggested that the prevalence of frailty in senior citizens ranges from $4.3 \%$ [21] to $37.2 \%$ [22]. Such wide deviation is mainly due to variations in the populations under study or differences in the frailty evaluation tools. The 2012 study by Collard et al. [23] of
61500 elderly individuals in the USA, Canada, and Europe found that $10.7 \%$ were frail. The study included subjects from both rural and urban areas. Korean studies were not included the study. Collard et al. [23] also used diverse tools such as the Fried Frailty Index and the Study of Osteoporotic Fractures index, rather than the Kaigo-Yobo tool used in this study. When reviewing the studies which estimated the prevalence of frailty and aging-related health conditions in older Koreans in rural communities, Jang et al. [24] found that $17.4 \%$ of the subjects were frail, while that figure was $27.5 \%$ in the 2016 study by Jung et al. [25]. The results may vary depending on the region where the subjects live. The studies conducted by Jang et al. [24] and Jung et al. [25] used the same populations, but the results of those studies were inconsistent due to the use of different evaluation tools (17.4\% when evaluated with the Fried Frailty Index and $27.5 \%$ with the Korean Frailty Index). Murayama et al. [13] in 2012 found the prevalence of frailty to be $10.6 \%$ using the same tool as this study. Murayama et al. [13] targeted dwellers of satellite cities, which may explain why the results were inconsistent with the estimates of this study. The prevalence of frailty found in this study may have been higher than that found in other studies due to the region's sociodemographic and geographical characteristics, as well as the characteristics of the agricultural industry of the region. The subjects of this study mostly made cash crops grown in greenhouses; this involves relatively uncomfortable working positions and intensive labor, leading to a higher prevalence of musculoskeletal conditions and a lower health-related quality of life [26]. Such variation in the results is why careful interpretation of outcomes is crucial when analyzing studies conducted in rural regions.

The results suggested that low perceived neighborhood walkability and high environmental pollution that interferes with a walkable neighborhood environment are associated with frailty. These results align with existing studies, which 
suggested that poor land use mix, poor connectivity between sidewalks and public roads, as well as heavy traffic that affects pedestrians, led to reduced activities of daily living for the residents in the community [27], and that residents living in walkable communities had better self-rated health. Our results are also in line with studies that suggested a higher health-related quality of life among residents in neighborhoods with high accessibility to stores and parks [28].

Grip strength, a covariate in this study, is also used as a criterion for frailty. The Cardiovascular Health Study index, one of the most commonly used measurement tools of frailty, includes grip strength as 1 of its 5 criteria for frailty, which are weight loss, reduced vitality, reduced PA, reduced walking speed, and reduced grip strength [29]. Grip strength is used as a proxy for reduced muscle strength and sarcopenia in many studies [19], as it represents reduced physical functionality and frailty [30]. In other words, grip strength is often a measurement of physical frailty. However, this study used grip strength as an index for sarcopenia, not frailty itself. While many tools that measure frailty focus on physical frailty, the Kaigo-Yobo Checklist not only measures physical frailty, but also takes into account psychological and social frailty. Grip strength was adjusted to confirm whether walkability and environmental pollution affected psychological and social frailty, as well as physical frailty.

Additionally, a stratified analysis was performed to assess the difference between the 2 subject regions, although the regions have similar agricultural environments. The correlations between perceived neighborhood walkability and environmental pollution and frailty and the correlations between perceived neighborhood environmental pollution and frailty were similar across both regions with regard to size and direction, while the latter correlation was not statistically meaningful within a single location. Residents who are geographically closer together are likely to share the same roads and have similar results on objective indices of walkability and environmental pollution. Additional research is needed to confirm this geographical data.

The mechanism by which perceived neighborhood walkability and environmental pollution affect frailty can be explained in terms of physical frailty, psychological frailty, and social frailty. Regarding physical frailty, neighborhoods with a walkable environment and less environmental pollution allow residents to be more physically active, as they can more easily take a walk or go on foot rather than other transportations [7]. These factors also allow senior citizens to maintain mobility [31]. Additionally, they help prevent physical frailty as well as the development of diseases such as cardiovascular diseases, type 2 diabetes, osteoporosis, and cancer; the latter is accomplished through the enhancement of muscle strength, endurance, sense of balance, and sensory functions [32]. With respect to psychological frailty, mental health can be promoted through the simple maintenance of physical functioning by preventing physical frailty [33]. Moreover, aesthetically pleasing and clean streets, pedestrian-friendly sidewalks, and accessibility to various services can help decrease depression among residents [34]. Residents from neighborhoods with a worse built environment are $35 \%$ to $64 \%$ more likely to be depressed than residents from neighborhoods with a better built environment [35]. Another study suggested that a poor built environment in communities leads to more mental and social stress, resulting in higher alcohol consumption [36]. Enjoying PAs such as walking in new environments or spending time in nature outside of the home helps prevent psychological frailty among the elderly by providing emotional stability, decreasing depression, and promoting vitality [32]. Finally, with respect to social frailty, residents in neighborhoods with many places to walk are more likely to socialize and receive community support by being able to easily meet neighbors and find walking companions. The act of becoming close with neighbors leads to better socialization and connectivity [37]. Residents in communities with longer stretches of sidewalk tend to walk more, have more trust in their neighbors, and have more social capital [38]. High perceived safety in communities, which can be promoted by factors such as improved lighting, can also increase the social capital of the residents and potentially decrease social frailty [39].

The possibility of reverse causation should be considered, given that this is a cross-sectional study. Frail senior citizens may perceive their neighborhood environment negatively, which leads to decreased walking and PA, which in turn leads to a higher prevalence of frailty. Interventions to improve local environmental conditions when possible are essential to prevent this vicious cycle and to decrease frailty. Another potential example of reverse causation is that people who engage in frequent walking and other PA outside the home may perceive low walkability in their neighborhood better than those who do not. Longitudinal studies on a larger scale will be needed to confirm the exact nature of causation between environmental factors and frailty.

The limitations of this study are as follows. First, proving causation between perceived neighborhood walkability and 
environmental pollution, and frailty is difficult due to the cross-sectional design of the study. Second, the study was conducted in 2 towns in the Gyeongnam Province and therefore does not represent the entire rural population of Korea. Further research on rural regions with different industries, populations, and environmental structures must be carried out in order to provide a more generalizable conclusion. Research on foreign residents should also be conducted, considering the high proportion of non-Korean residents in rural regions. Third, this study did not include an objective evaluation of the environmental factors of the region, as its results are based on perceived neighborhood walkability and environmental pollution, not on data from community audits or geographic information systems. Studies exploring the correlation between one's regional environment and PA showed varying outcomes depending on whether the environmental factors were measured objectively or subjectively. The correlation tended to be higher when using subjective measurements than objective measurements, but this does not imply that one method is superior to another method [40]. Although this study is about frailty rather than PA, it is possible that the subjective nature of the participants' assessment of the local environment may have exaggerated the outcomes. Fourth, the validity of the tools used to assess perceived neighborhood walkability and environmental pollution should be verified. The survey questions included some questions that have commonly been used to evaluate walkability, but the validity of the adjusted or revised questions should be evaluated to determine whether they were appropriate for evaluating the walkability of rural areas for elderly residents. Moreover, a new tool suitable for rural environments should be developed rather than just adopting the existing tools, which mostly target urban environments.

Despite the limitations, this study is meaningful in that it was the first exploratory study to confirm the correlation between local environmental factors and frailty, given that there has been little research on environmental factors as determinants of frailty. Frailty was observed in $35.5 \%$ of the rural elderly population under the study, and low perceived neighborhood walkability and high environmental pollution corresponded to a higher prevalence of frailty. These results are consistent with previous studies. Further research is needed to understand the characteristics and extent of frailty by community, as are intervention and longitudinal studies focusing on local environmental factors.
As the population of Korea continues to age, environmental factors is expected to become more important in the promotion of healthy aging. Rural towns have a higher proportion of elderly population than urban areas, which is why more attention should be paid to promoting physical, psychological, and social health in order to prevent frailty and enhance elderly health in rural areas as well as modifiable poor built environments.

\section{SUPPLEMENTAL MATERIALS}

Supplemental materials are available at https://doi.org/10. 3961/jpmph.19.166.

\section{CONFLICT OF INTEREST}

The authors have no conflicts of interest associated with the material presented in this paper.

\section{ACKNOWLEDGEMENTS}

This study was funded by the Korea Centers for Disease Control and Prevention (Research Project No. 2018-30).

\section{AUTHOR CONTRIBUTIONS}

Conceptualization: KSP, MJK, BGJ, JRK. Data curation: MJK, YSC, ARS. Formal analysis: MJK, SHS, KSP. Funding acquisition: KSP. Methodology: KSP, BGJ, YSK, JRK, YSC, BKK, GYL, ARS. Project administration: SHS, ARS, BKK, MJK. Visualization: MJK, SHS, KSP. Writing - original draft: MJK, JHK, KSP, ARS, BKK, GYL, YSC. Writing - review \& editing: MJK, JHK, KSP, SHS, JRK, YSK, BGJ.

\section{ORCID}

Mi-Ji Kim https://orcid.org/0000-0002-8646-832X Sung-Hyo Seo https://orcid.org/0000-0003-3327-5425 Ae-Rim Seo https://orcid.org/0000-0002-9444-5953 Bo-Kyoung Kim https://orcid.org/0000-0002-1926-9785 Gyeong-Ye Lee https://orcid.org/0000-0002-3217-7324 Yeun-Soon Choi https://orcid.org/0000-0002-6200-2875 Jin-Hwan Kim https://orcid.org/0000-0001-8229-9613 Jang-Rak Kim https://orcid.org/0000-0002-8191-5071 Yune-Sik Kang https://orcid.org/0000-0002-3404-279X Baek-Geun Jeong https://orcid.org/0000-0002-1655-5436 Ki-Soo Park https://orcid.org/0000-0001-5571-3639 


\section{REFERENCES}

1. Korean Statistical Information Service. Statistics on the aged in 2018 [cited 2018 Sep 27]. Available from: http://kosis.kr/ publication/publicationWord.do (Korean).

2. Gobbens RJ, Luijkx KG, Wijnen-Sponselee MT, Schols JM. Towards an integral conceptual model of frailty. J Nutr Health Aging 2010;14(3):175-181.

3. Kim DH, Arai H. Social frailty. Korean J Clin Geriatr 2015;16(2): 44-49 (Korean).

4. Gobbens RJ, van Assen MA, Luijkx KG, Wijnen-Sponselee MT, Schols JM. Determinants of frailty. J Am Med Dir Assoc 2010; 11(5):356-364.

5. Topinková E. Aging, disability and frailty. Ann Nutr Metab 2008; 52 Suppl 1:6-11.

6. Xue QL, Fried LP, Glass TA, Laffan A, Chaves PH. Life-space constriction, development of frailty, and the competing risk of mortality: the Women's Health And Aging Study I. Am J Epidemiol 2008;167(2):240-248

7. Barnett DW, Barnett A, Nathan A, Van Cauwenberg J, Cerin E; Council on Environment and Physical Activity (CEPA) - Older Adults Working Group. Built environmental correlates of older adults' total physical activity and walking: a systematic review and meta-analysis. Int J Behav Nutr Phys Act 2017;14(1):103.

8. Mahmood A, Chaudhury H, Michael YL, Campo M, Hay K, Sarte A. A photovoice documentation of the role of neighborhood physical and social environments in older adults' physical activity in two metropolitan areas in North America. Soc Sci Med 2012;74(8):1180-1192.

9. Grant MJ, Booth A. A typology of reviews: an analysis of 14 review types and associated methodologies. Health Info Libr J 2009;26(2):91-108.

10. Brunekreef B, Holgate ST. Air pollution and health. Lancet 2002; 360(9341):1233-1242.

11. Gent JF, Triche EW, Holford TR, Belanger K, Bracken MB, Beckett WS, et al. Association of low-level ozone and fine particles with respiratory symptoms in children with asthma. JAMA 2003; 290(14):1859-1867.

12. Hwang HS, Yoon JL, Park BJ, Choi HR, Kwon IS, Shinkai S, et al. The validity and reliability of the Kaigo-Yobo checklist in Korean elderly. J Korean Geriatr Soc 2012;16(3):121-132 (Korean).

13. Murayama H, Nishi M, Shimizu Y, Kim MJ, Yoshida H, Amano H, et al. The Hatoyama Cohort Study: design and profile of participants at baseline. J Epidemiol 2012;22(6):551-558.

14. Saelens BE, Sallis JF, Black JB, Chen D. Neighborhood-based differences in physical activity: an environment scale evaluation. Am J Public Health 2003;93(9):1552-1558.

15. Heart Foundation. Neighbourhood walkability checklist. How walkable is your community? [cited 2019 Jun 1]. Available from: https://www.heartfoundation.org.au/images/uploads/ main/Active_living/Neighbourhood-walkability-checklist.pdf.

16. Sallis JF, Kerr J, Carlson JA, Norman GJ, Saelens BE, Durant N, et al. Evaluating a brief self-report measure of neighborhood environments for physical activity research and surveillance: Physical Activity Neighborhood Environment Scale (PANES). J Phys Act Health 2010;7(4):533-540.

17. Hanibuchi T, Nakaya T, Yonejima M, Honjo K. Perceived and objective measures of neighborhood walkability and physical activity among adults in Japan: a multilevel analysis of a nationally representative sample. Int J Environ Res Public Health 2015;12(10):13350-13364.

18. Ministry of Health and Welfare. 2018 median income and criteria for selection of medical benefit and national minimum [cited 2017 Aug 18]. Available from: http://www.mohw.go.kr/ react/jb/sjb0406vw.jsp?PAR_MENU_ID=03\&MENU_ID=030406 \&CONT_SEQ $=341140$ (Korean).

19. Yoo Jl, Choi H, Ha YC. Mean hand grip strength and cut-off value for sarcopenia in Korean adults using KNHANES VI. J Korean Med Sci 2017;32(5):868-872.

20. Chen LK, Liu LK, Woo J, Assantachai P, Auyeung TW, Bahyah $\mathrm{KS}$, et al. Sarcopenia in Asia: consensus report of the Asian Working Group for Sarcopenia. J Am Med Dir Assoc 2014; 15(2):95-101.

21. Al Snih S, Graham JE, Ray LA, Samper-Ternent R, Markides KS, Ottenbacher KJ. Frailty and incidence of activities of daily living disability among older Mexican Americans. J Rehabil Med 2009;41(11):892-897.

22. Aguilar-Navarro SG, Amieva H, Gutiérrez-Robledo LM, AvilaFunes JA. Frailty among Mexican community-dwelling elderly: a story told 11 years later. The Mexican Health and Aging Study. Salud Publica Mex 2015;57 Suppl 1:S62-S69.

23. Collard RM, Boter H, Schoevers RA, Oude Voshaar RC. Prevalence of frailty in community-dwelling older persons: a systematic review. J Am Geriatr Soc 2012;60(8):1487-1492.

24. Jang IY, Jung HW, Lee CK, Lee YS, Kim KI, Kim KW, et al. Rural and urban disparities in frailty and aging-related health conditions in Korea. J Am Geriatr Soc 2016;64(4):908-911.

25. Jung HW, Jang IY, Lee YS, Lee CK, Cho El, Kang WY, et al. Prevalence of frailty and aging-related health conditions in older Koreans in rural communities: a cross-sectional analysis of the 
aging study of Pyeongchang rural area. J Korean Med Sci 2016; 31(3):345-352.

26. Chong BH, Yang CY, Oh GJ. The difference of subjective symptoms of musculoskeletal disorders and health related quality of life in vinylhouse and non-vinylhouse farmers. J Korean Soc Saf 2010;25(4):68-75 (Korean).

27. Clarke P, George LK. Understanding and addressing the challenges of disability: the role of the built environment. Am J Epidemiol 2005;95(11):1933-1939.

28. Renalds A, Smith TH, Hale PJ. A systematic review of built environment and health. Fam Community Health 2010;33(1): 68-78.

29. Fried LP, Tangen CM, Walston J, Newman AB, Hirsch C, Gottdiener J, et al. Frailty in older adults: evidence for a phenotype. J Gerontol A Biol Sci Med Sci 2001;56(3):M146-M156.

30. Cesari M, Landi F, Vellas B, Bernabei R, Marzetti E. Sarcopenia and physical frailty: two sides of the same coin. Front Aging Neurosci 2014;6:192.

31. Clarke P, Ailshire JA, Bader M, Morenoff JD, House JS. Mobility disability and the urban built environment. Am J Epidemiol 2008;168(5):506-513.

32. Mosallanezhad Z, Salavati M, Sotoudeh GR, Nilsson Wikmar L, Frändin K. Walking habits and health-related factors in 75year-old Iranian women and men. Arch Gerontol Geriatr 2014; 58(3):320-326.

33. Zhao Y, Chung PK. Neighborhood environment walkability and health-related quality of life among older adults in Hong Kong. Arch Gerontol Geriatr 2017;73:182-186.

34. Rautio N, Filatova S, Lehtiniemi H, Miettunen J. Living environment and its relationship to depressive mood: a systematic review. Int J Soc Psychiatry 2018;64(1):92-103.

35. Galea S, Ahern J, Rudenstine S, Wallace Z, Vlahov D. Urban built environment and depression: a multilevel analysis. J Epidemiol Community Health 2005;59(10):822-827.

36. Bernstein KT, Galea S, Ahern J, Tracy M, Vlahov D. The built environment and alcohol consumption in urban neighborhoods. Drug Alcohol Depend 2007;91(2-3):244-252.

37. Leyden KM. Social capital and the built environment: the importance of walkable neighborhoods. Am J Public Health 2003; 93(9):1546-1551.

38. Wood L, Shannon T, Bulsara M, Pikora T, McCormack G, GilesCorti B. The anatomy of the safe and social suburb: an exploratory study of the built environment, social capital and residents' perceptions of safety. Health Place 2008;14(1):15-31.

39. Araya R, Dunstan F, Playle R, Thomas H, Palmer S, Lewis G. Perceptions of social capital and the built environment and mental health. Soc Sci Med 2006;62(12):3072-3083.

40. Cerin E, Sit CH, Cheung MC, Ho SY, Lee LC, Chan WM. Reliable and valid NEWS for Chinese seniors: measuring perceived neighborhood attributes related to walking. Int J Behav Nutr Phys Act 2010;7:84. 